\title{
EP-37
}

\section{Impact of anatomical liver resection for hepatocellular carcinoma: A systematic review and meta-analysis}

\author{
SeongWook SHIN, Tae-Seok KIM*
}

Division of Hepatobiliary \& Pancreatic Surgery, Department of Surgery, Dongsan Medical Center, Keimyung University School of Medicine, Daegu, Korea

Introduction: Although several retrospective studies comparing anatomical liver resection (AR) and non-anatomical liver resection (NAR) for hepatocellular carcinoma (HCC) have been published, the efficacy of anatomic liver resection for HCC remains unclear. This study aimed to compare the oncologic and surgical outcomes of AR and NAR for HCC by performing meta-analysis of published studies.

Methods: We systemically reviewed the MEDLINE, Embase, and Cochrane Library databases for propensity score matching (PSM) studies compared AR and NAR for HCC. The primary outcome was overall survival (OS) and recurrence free survival (RFS). Secondary outcomes were recurrence patterns and peri-operative outcomes.

Results: A total of 19 PSM studies were included. AR was superior to NAR in 3- and 5-year OS (3-year OS hazard ratio [HR]: 0.79, 95\% confidence interval [CI]: 0.68-0.91, $p=0.001$; 5-year OS HR: 0.83, 95\% CI: $0.71-0.95, p=0.009)$. AR showed a significantly better 1-, 3-, and 5-year RFS than NAR with low local recurrence rate, intra- and extra-hepatic recurrence (1-year RFS HR: 0.79, 95\% CI: 0.69-0.90, $p=0.0005$; 3-year RFS HR: 0.81, 95\% CI: 0.75-0.87; $p=0.00001$; 5-year RFS HR: 0.82, 95\% CI: 0.76-0.88, $p<0.00001)$. In subgroup analyses about tumor with diameter less than $5 \mathrm{~cm}$ and with microscopic cancer spread, RFS in AR group was significantly better than NAR. However, AR showed comparable 3- and 5-year RFS with NAR group in patients with cirrhotic liver. Also, post-operative overall complications including biliary leakage and hepatic failure were similar between two groups.

Conclusions: Our meta-analysis revealed that AR showed better OS and RFS with low local recurrence than NAR especially patients with tumor diameter less than $5 \mathrm{~cm}$ and non-cirrhotic liver. 